\title{
The single institutional outcome of postoperative radiotherapy and concurrent chemoradiotherapy in resected non-small cell lung cancer
}

\author{
Hyo Chun Lee, MD1, Yeon Sil Kim, MD1, Se Jin Oh, MD1, Yun Hee Lee, MD¹, Dong Soo Lee, MD², \\ Jin Ho Song, MD', Jin Hyung Kang, MD², Jae Kil Park, MD³ \\ Departments of ${ }^{1}$ Radiation Oncology, ${ }^{2}$ Medical Oncology, and ${ }^{3}$ Thoracic Surgery, Seoul St. Mary's Hospital, \\ The Catholic University of Korea College of Medicine, Seoul, Korea
}

\begin{abstract}
Purpose: This study was conducted to observe the outcomes of postoperative radiotherapy (PORT) with or without concurrent chemotherapy in resected non-small cell lung cancer (NSCLC) in single institution.

Materials and Methods: From 2002 to 2013, 78 patients diagnosed with NSCLC after curative resection were treated with radiotherapy alone ( $R T, n=48$ ) or concurrent chemoradiation (CCRT, $n=30$ ). The indications of adjuvant radiation therapy were N2 node positive $(n=31)$, close or involved resection margin $(n=28)$, or gross residual disease due to incomplete resection $(n=19)$. The median radiation dose was $57.6 \mathrm{~Gy}$ (range, 29.9 to $66 \mathrm{~Gy}$ ).

Results: Median survival time was 33.7 months (range, 4.4 to 140.3 months). The 5 -year overall survival (OS) rate was $49.5 \%$ (RT $46 \%$ vs. CCRT 55.2\%; $p=0.731$ ). The 3 -year disease-free survival rate was $45.5 \%$ (RT $39.4 \%$ vs. CCRT $55.3 \% ; p=0.130$ ). The 3 -year local control rate was $68.1 \%$ (RT $64.4 \%$ vs. CCRT $77.7 \% ; p=0.165$ ). The 3 -year DMFS rate was $56.1 \%$ (RT $52.6 \%$ vs. CCRT $61.7 \% ; p$ $=0.314$ ). In multivariate analysis, age $\geq 66$ years and pathologic stage III were significant poor prognostic factors for OS. Treatment failure occurred in 40 patients. Four patients had radiologically confirmed grade 3 radiation pneumonitis.

Conclusion: In NSCLC, adjuvant RT or CCRT after curative surgery is a safe and feasible modality of treatment. OS gain was seen in patients less than 66 years. Postoperative CCRT showed a propensity of achieving better local control and improved disease-free survival compared to RT alone according to our data.
\end{abstract}

Keywords: Non-small-cell lung carcinoma, Radiotherapy, Chemoradiotherapy

\section{Introduction}

Lung cancer is the fifth common cancer and the first leading cause of cancer death in Korea. Surgery is the treatment of choice in stages I and II non-small cell lung cancer (NSCLC), and could be considered in selected stage III patients. However, the overall survival (OS) rate has not been satisfactory even though the tumor had been completely resected [1]. Locoregional recurrence after resection of NSCLC is common, occurring in approximately $20 \%$ of patients with stage I disease and up to $50 \%$ of patients with stage III disease [26]. The 5 -year relative survival rate of NSCLC has increased, rating $17.5 \%$ in $2006-2010$ compared to $10.4 \%$ in 1993-1995 due to the development of new treatment technology and

Received 2 May 2014, Revised 30 June 2014, Accepted 8 August 2014.

Correspondence: Yeon Sil Kim, MD, Department of Radiation Oncology, Seoul St. Mary's Hospital, 222 Banpo-daero, Seocho-gu, Seoul 137-701, Korea. Tel: +82-2-2258-6259, Fax: +82-2-592-1523, E-mail: yeonkim7@catholic.ac.kr

(c) This is an Open Access article distributed under the terms of the Creative Commons Attribution Non-Commercial License (http://creativecommons.org/ licenses/by-nc/3.0/) which permits unrestricted non-commercial use, distribution, and reproduction in any medium, provided the original work is properly cited.

www.e-roj.org 
chemotherapeutic agents [7]. Postoperative radiotherapy (PORT) has been investigated as a treatment option to reduce locoregional recurrence and furthermore, improve survival. In SEER analysis, PORT improved OS in N2 node positive patients, but reduced survival in NO and N1 patients [8]. The results of randomized clinical trials in the modern radiotherapy era are currently not available; hence, the role of PORT remains controversial.

The role of chemoradiation in postoperative setting has been explored with positive outcomes in favor of adjuvant chemotherapy. In ECOG 3590 trial, the addition of concurrent chemotherapy showed no benefit in local recurrence or survival in stages II and IIIA NSCLC [9]. By contrast, phase II study conducted by RTOG concluded that concurrent chemoradiation (CCRT) may indicate improved OS and progression-free survival in the same subset of patients. [10]. However, there are concerns about adjuvant chemoradiation causing additional toxicity without survival gain.

This study was designed to determine the outcomes of RT alone and CCRT in resected NSCLC, as well as to investigate whether CCRT shows more positive impact on the treatment outcome compared to RT alone. Factors associated with OS, disease-free survival (DFS), local control (LC), and distant metastasis free-survival (DMFS) were analyzed as well.

\section{Materials and Methods}

\section{Patients}

The medical records of 80 patients with NSCLC who had undergone curative surgery and PORT with or without concurrent chemotherapy at our institution, from 2002 to 2013, were reviewed retrospectively. Two patients were excluded from the study because both patients' final pathologic specimens were found to be mixed small cell and non-small cell carcinoma. Thus, the remaining 78 patients were examined in this study. There was no distant metastasis (MO) in our patients.

The characteristics of the patients and their tumors are shown in Table 1. The median age of all patients was 65.5 years (range, 44 to 79 years). More patients aged $\geq 66$ years were allocated in the RT arm $(n=29)$ than CCRT arm $(n=10 ; p=0.02)$. The CCRT arm had more patients with better performance status ( $p$ $=0.016$ ). The patients were subdivided into stage I-II and stage III. There were more stage I-II patients in the RT arm $(n=30)$ than the CCRT arm $(n=7 ; p=0.001)$. Consequently, the CCRT arm included more stage III patients, which was 56\% of them.
Other characteristics, such as tumor histology, grade, and lymphovascular invasion, showed no difference between the treatment arms. This study was approved by the Institutional Review Boards of our institution.

\section{Treatment}

1) Surgery: All patients underwent curative thoracic surgery. Surgical approaches were decided based on the tumor extent and the involved lymph node stations. There were 59 cases (75\%) of lobectomy, 5 cases (6.4\%) of pneumonectomy, and 13 cases (16.7\%) of wedge resection or segmentectomy. One patient's surgical approach was unidentified. Mediastinal lymph node dissection was performed on 72 patients, and among them, N2 nodes of 65 patients were surgically confirmed. In six patients, their mediastinal lymph nodes could not be surgically assessed because most of them were ineligible for extended surgery due to medical conditions, such as old age. The clinical stage for all 6 patients was NO. Both clinical and surgical staging were based on the 7th edition of the American Joint Committee on Cancer (AJCC) staging system.

2) Radiotherapy: External beam radiation therapy was delivered with 6 or 10 MV photons using a linear accelerator. Contrast-enhanced CT was used to generate the treatment plans. Patients were in supine position and both arms were placed over the head. The treatment position was fixed by customized vacuumed cushions. The radiation field was designed to cover the entire mediastinum including the uninvolved nodal stations ( $n=26$ ) or primary tumor bed and nearest mediastinal and hilar lymph node stations ( $n=52)$, based on the tumor extent and lymph node status. In case of upper and middle lobe tumors, lower mediastinum below the subcarinal level was excluded from the radiation field. Likewise, the highest mediastinum was excluded in lower lobe tumors. Three-dimensional conformal radiotherapy (3D-CRT) plan with inhomogeneity correction was carried out in all patients. Since 2009, 4D simulation CT was employed in order to apply the tumor movement in generation of planning target volume.

Adjuvant RT indications included N2 nodal metastasis ( = 31), involved (R1) or tumor within $1 \mathrm{~cm}$ (RO) of resection margins ( $n=28)$, or gross residual tumor due to incomplete (R2) resection $(n=19)$. This category showed difference between the treatment arms, such that more patients with microscopic or gross residual disease were in the RT arm ( $p=$ 0.031). 
Table 1. Patient and tumor characteristics

\begin{tabular}{|c|c|c|c|c|}
\hline Characteristic & Total $(n=78)$ & $\mathrm{RT}(\mathrm{n}=48)$ & CCRT $(n=30)$ & $p$-value \\
\hline Age (yr) & $65.5(44-79)$ & $68(48-79)$ & $62(44-74)$ & 0.020 \\
\hline$\geq 66$ & $39(50.0)$ & $29(74.0)$ & $10(26.0)$ & \\
\hline$<66$ & $39(50.0)$ & $19(49.0)$ & $20(51.0)$ & \\
\hline Sex & & & & 0.795 \\
\hline Male & $61(78.0)$ & $38(62.0)$ & $23(38.0)$ & \\
\hline Female & $17(22.0)$ & $10(59.0)$ & $7(41.0)$ & \\
\hline ECOG performance status & & & & 0.016 \\
\hline 0 & $18(23.0)$ & $6(33.0)$ & $12(67.0)$ & \\
\hline 1 & $58(74.0)$ & $41(71.0)$ & $17(29.0)$ & \\
\hline 2 & $2(3.0)$ & $1(50.0)$ & $1(50.0)$ & \\
\hline Histology & & & & 0.244 \\
\hline Adenocarcinoma & $39(50.0)$ & $21(55.0)$ & $17(45.0)$ & \\
\hline $\mathrm{SqCC}$ & $37(47.0)$ & $25(68.0)$ & $12(32.0)$ & \\
\hline Large cell & $1(1.0)$ & $1(100)$ & $0(0)$ & \\
\hline Other & $1(1.0)$ & $1(50.0)$ & $1(50.0)$ & \\
\hline Grade & & & & 0.108 \\
\hline Well & $4(5.0)$ & $1(25.0)$ & $3(75.0)$ & \\
\hline Moderate & $52(67.0)$ & $36(69.0)$ & $16(31.0)$ & \\
\hline Poor & $18(23.0)$ & $8(44.0)$ & $10(56.0)$ & \\
\hline Undetermined & $4(5.0)$ & $3(75.0)$ & $1(25.0)$ & \\
\hline Resection margin & & & & 0.380 \\
\hline Negative $(\geq 1 \mathrm{~cm})$ & 38 (49.0) & 19 (53.0) & $19(47.0)$ & \\
\hline Close $(<1 \mathrm{~cm})$ & $31(40.0)$ & $22(67.0)$ & $9(33.0)$ & \\
\hline Positive & $7(9.0)$ & $5(71.0)$ & $2(29.0)$ & \\
\hline Unknown & $2(3.0)$ & $2(100)$ & $0(0)$ & \\
\hline AJCC stage & & & & 0.001 \\
\hline$|-| \mid$ & $37(47.0)$ & 30 (81.0) & $7(19.0)$ & \\
\hline III & 41 (53.0) & 18 (44.0) & $23(56.0)$ & \\
\hline рт & & & & 0.071 \\
\hline 1 & $23(29.0)$ & $13(57.0)$ & $10(43.0)$ & \\
\hline 2 & $31(40.0)$ & $23(74.0)$ & $8(26.0)$ & \\
\hline 3 & 16 (21.0) & $10(63.0)$ & $6(37.0)$ & \\
\hline 4 & $8(10.0)$ & $2(25.0)$ & $6(75.0)$ & \\
\hline $\mathrm{pN}$ & & & & 0.050 \\
\hline 0 & $26(33.0)$ & $20(77.0)$ & $6(23.0)$ & \\
\hline 1 & $13(17.0)$ & $8(62.0)$ & $5(38.0)$ & \\
\hline 2 & $31(40.0)$ & 15 (48.0) & $16(52.0)$ & \\
\hline 3 & $2(3.0)$ & $0(0)$ & $2(100)$ & \\
\hline x & $6(8.0)$ & $5(83.0)$ & $1(17.0)$ & \\
\hline Lymphatic invasion & & & & 0.300 \\
\hline Negative & $23(29.0)$ & $16(70.0)$ & $7(30.0)$ & \\
\hline Positive & $51(65.0)$ & $28(57.0)$ & $23(43.0)$ & \\
\hline Unknown & $4(5.0)$ & $4(100)$ & $0(0)$ & \\
\hline Vascular invasion & & & & 0.358 \\
\hline Negative & $60(77.0)$ & $37(62.0)$ & $23(38.0)$ & \\
\hline Positive & $12(15.0)$ & 8 (73.0) & $3(27.0)$ & \\
\hline Unknown & $6(8.0)$ & $3(43.0)$ & $4(57.0)$ & \\
\hline
\end{tabular}

Values are presented as median (range) or number of patients (\%).

RT, radiotherapy; CCRT, concurrent chemoradiation; ECOG, Eastern Cooperative Oncology Group; SqCC, squamous cell carcinoma; AJCC, American Joint Committee on Cancer. 
Table 2. Treatment related factors by the treatment arms

\begin{tabular}{|c|c|c|c|c|}
\hline & Total $(n=78)$ & $\mathrm{RT}(\mathrm{n}=48)$ & $\operatorname{CCRT}(n=30)$ & $p$-value \\
\hline RT indication & & & & 0.031 \\
\hline N2 node (+) & $31(40.0)$ & $14(45.0)$ & $17(55.0)$ & \\
\hline Close or (+) RM & $28(36.0)$ & $22(79.0)$ & $6(21.0)$ & \\
\hline Residual disease & $19(24.0)$ & $12(63.0)$ & $7(37.0)$ & \\
\hline Surgery type & & & & 0.838 \\
\hline Lobectomy & $59(76.0)$ & $37(63.0)$ & $22(38.0)$ & \\
\hline Pneumonectomy & $4(5.0)$ & $3(60.0)$ & $2(40.0)$ & \\
\hline Wedge resection or segmentectomy & $13(17.0)$ & $7(54.0)$ & $6(46.0)$ & \\
\hline RT field & & & & 0.139 \\
\hline Surgical bed only & $52(67.0)$ & $35(67.0)$ & $17(33.0)$ & \\
\hline Entire mediastinum & $26(33.0)$ & $13(50.0)$ & $13(50.0)$ & \\
\hline RT dose (Gy) & & & & 0.001 \\
\hline$\geq 57.6$ & $40(51.0)$ & $17(43.0)$ & $23(58.0)$ & \\
\hline$<57.6$ & $38(49.0)$ & 31 (82.0) & $7(18.0)$ & \\
\hline
\end{tabular}

Values are presented as number of patients (\%).

$\mathrm{RT}$, radiotherapy; CCRT, concurrent chemoradiation; $\mathrm{RM}$, resection margin.

The median total dose for all patients was 57.6 Gy ranging from 29.9 to $66 \mathrm{~Gy}$. The median dose of RT arm and CCRT arm were 55.8 and $59.4 \mathrm{~Gy}$, respectively. More patients in CCRT arm received $\geq 57.6$ Gy compared with $R T$ arm $(p=0.001)$ (Table 2 ). One patient in RT arm whose irradiation dose was only $29.9 \mathrm{~Gy}$ showed low compliance to the treatment and did not receive the initially planned dose. In another patient in RT arm, RT was interrupted at $45 \mathrm{~Gy}$ out of total dose of $55 \mathrm{~Gy}$, because malignant effusion developed. Rest of the patients received the planned radiation dose of more than $50 \mathrm{~Gy}$.

3) Chemotherapy: Concurrent chemoradiation was given to 30 patients. Among them, 17 patients were multiple N2 node positive, 6 patients had involved or close $(<1$ $\mathrm{cm}$ ) resection margin, and the remaining 7 patients were incompletely resected cases with gross residual disease. Various chemotherapy regimens were used-paclitaxel $(n=8)$, cisplatin $(n=10)$, docetaxel-cisplatin $(n=8)$, and paclitaxelcarboplatin $(n=2)$. Among the 29 patients who received adjuvant chemotherapy, 9 of them had their chemotherapy started before radiation and the other 20 sequentially after radiotherapy.

\section{Evaluation}

During radiotherapy, patients had weekly physical examination, complete blood count, and plain chest $X$-ray for the evaluation of acute toxicity. After radiotherapy, patients visited the clinic every 3 months for the first 2 years, and 6 months thereafter for surveillance of late complications and recurrence. Adverse effects of chemotherapy and radiotherapy were assessed using the National Cancer Institute Common Terminology Criteria for Adverse Events (v3.0) and RTOG Acute and Late Lung Morbidity Scoring Criteria for radiation pneumonitis. Incidence of toxicity grade $\geq 2$ was recorded.

\section{Statistical analysis}

Kaplan-Meier method was used for OS, DFS, LC, and DMFS rates. The prognostic value of patient age, performance status, histologic grade, lymphovascular invasion, resection margin status, pathologic stage, type of surgery, radiation field and dose, and systemic chemotherapy were analyzed. In univariate analysis, log-rank test was utilized to evaluate the association between each survival time and prognostic factors. For multivariate analysis, Cox proportional hazards regression model was employed to estimate the hazard ratio of the prognostic factors for OS, DFS, LC, and DMFS. Statistical significance was evaluated at 0.05 alpha levels.

\section{Results}

The median follow-up time for all patients was 25.9 months (range, 1.2 to 97.5 months) and 44.2 months (range, 15.9 to 137.4 months) for survivors. The median survival time was 33.7 months (range, 4.4 to 140.3 months). The 5 -year OS rate of all 78 patients was $49.5 \%$. The 5 -year OS rates were $46 \%$ for the RT arm and 55.2\% for the CCRT arm ( $p=0.731)$ (Fig. 
A

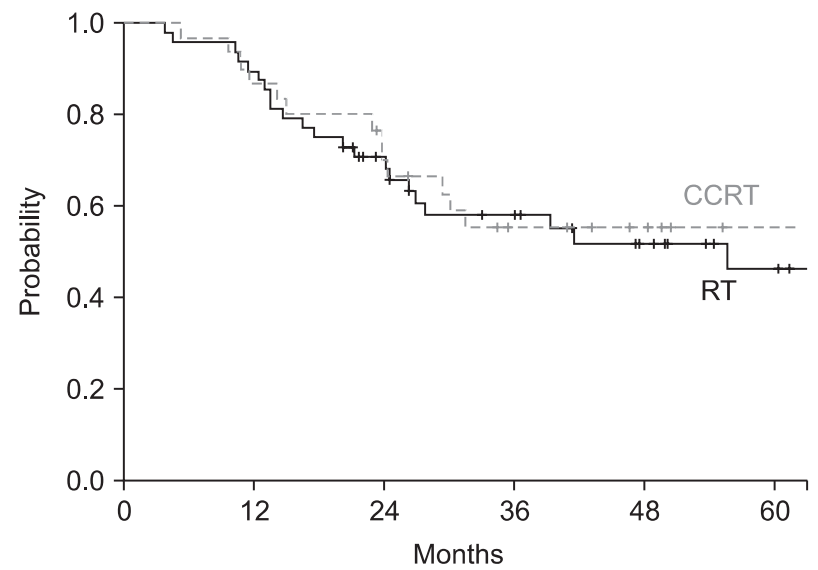

B

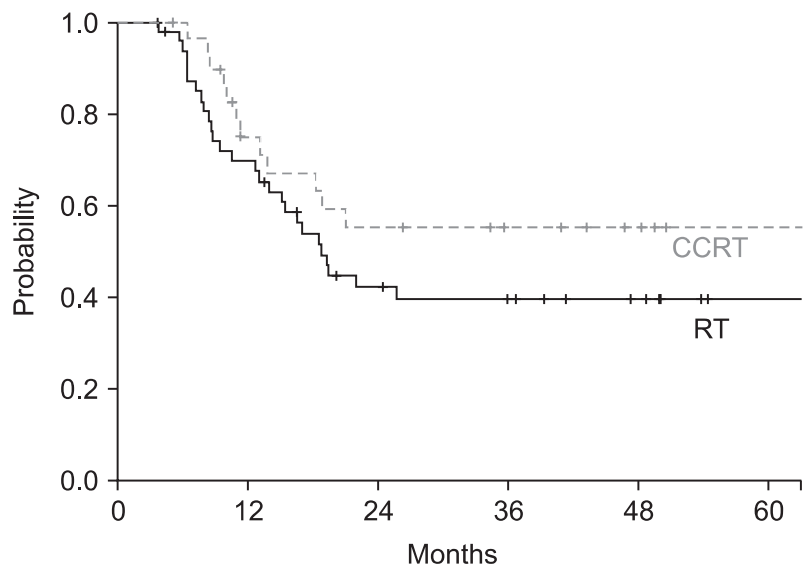

Fig. 1. Overall survival (A) and disease-free survival (B) of patients treated by radiotherapy (RT) and concurrent chemoradiation (CCRT).

A

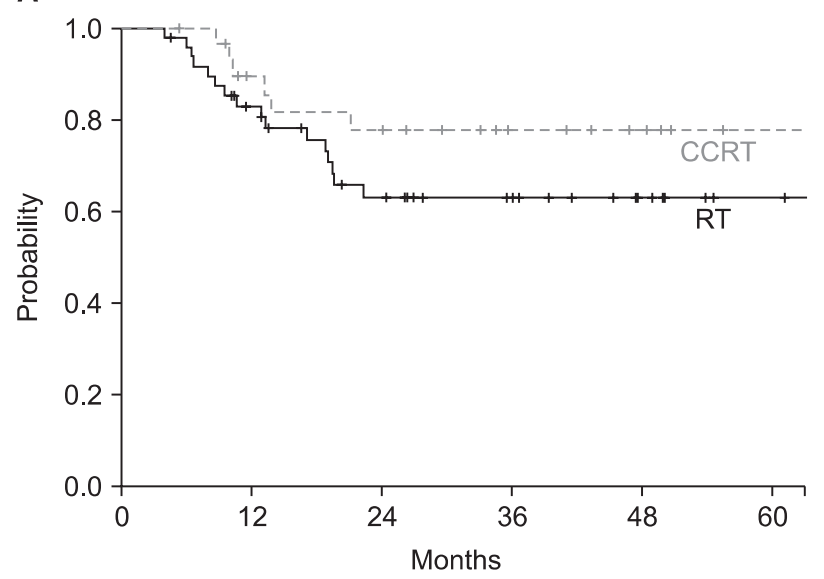

B

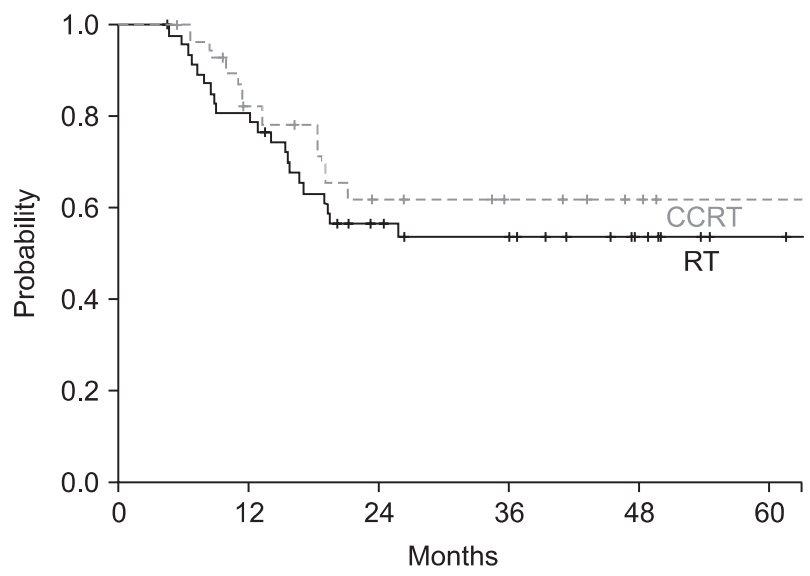

Fig. 2. Local control (A) and distant metastasis-free survival (B) of patients treated by radiotherapy (RT) and concurrent chemoradiation (CCRT).

1). The 3-year DFS in all patients, RT arm and CCRT arm were $45.5 \%, 39.4 \%$, and 55.3\%, respectively ( $p=0.130$ ) (Fig. 1). The 3-year LC rate was $68.1 \%$ for all patients, $64.4 \%$ for RT arm and $77.7 \%$ for CCRT arm ( $p=0.165)$ (Fig. 2). The DMFS at 3 -year for all patients was $56.1 \%$, the RT arm was $52.6 \%$, and the CCRT arm was 61.7\%, respectively ( $p=0.314)$ (Fig. 2$)$.

In univariate analysis, although CCRT arm had higher survival rate, the difference was not statistically significant compared to RT alone arm. Total radiation dose above 57.6 Gy and radiation field extended for elective mediastinal coverage showed better DFS and DMFS. Rest of the prognostic factors and their univariate analyses on OS, DFS, LC, and DMFS are summarized in Table 3.

Multiple factors significantly affecting survival in univariable tests were assessed using multivariable analyses. Age $<66$ years $(p<0.001)$ and pathologic stage III $(p=0.015)$ were found to be poor prognostic factors of OS. Higher total radiation dose was a significant factor for DFS and DMFS. The results of multivariate analyses are listed in Table 4.

Treatment failure occurred in 40 patients (51.3\%); 8 patients failed locoregionally (within the mediastinum), 18 patients distantly, and 14 patients both locoregionally and distantly. Distant metastasis sites were as follows: 11 in lung, 8 in brain, 7 in bone, 5 in liver, 5 in adrenal gland, 4 in non-regional lymph node, and 1 each in kidney and pericardial effusion. The pattern of recurrence by two treatment arms is listed in Table 5 . There was no statistical difference was seen between the arms. Table 6 demonstrates radiotherapy related toxicities. Acute and subacute radiation pneumonitis of grades 2 and 3 were seen in $6(7.7 \%)$ and $3(3.8 \%)$ patients, respectively. Among 
Table 3. Univariate analysis of prognostic factors

\begin{tabular}{|c|c|c|c|c|}
\hline \multirow{2}{*}{ Variable } & \multicolumn{4}{|c|}{$p$-value } \\
\hline & OS & DFS & $\mathrm{LC}$ & DMFS \\
\hline Age (<66 vs. $\geq 66$ yr) & 0.004 & 0.198 & 0.783 & 0.508 \\
\hline Sex (male vs. female) & 0.892 & 0.628 & 0.544 & 0.343 \\
\hline ECOG performance status (0 vs. 1, 2) & 0.896 & 0.951 & 0.790 & 0.310 \\
\hline Tumor grade & 0.008 & 0.028 & 0.062 & 0.084 \\
\hline Margin status & 0.113 & 0.106 & 0.190 & 0.679 \\
\hline Lymphatic invasion & 0.354 & 0.057 & 0.182 & 0.103 \\
\hline Vascular invasion & 0.500 & 0.071 & 0.081 & 0.041 \\
\hline Stage (I-II vs. III) & 0.228 & 0.033 & 0.135 & 0.036 \\
\hline No. of positive lymph node (<4 vs. $\geq 4)$ & 0.181 & 0.001 & 0.018 & 0.007 \\
\hline Treatment modality (RT vs. CCRT) & 0.731 & 0.130 & 0.165 & 0.314 \\
\hline Total radiation dose (<57.6 vs. $\geq 57.6$ Gy) & 0.847 & 0.041 & 0.088 & 0.023 \\
\hline Radiation field (tumor bed vs. mediastinum) & 0.168 & 0.004 & 0.067 & 0.015 \\
\hline
\end{tabular}

OS, overall survival; DFS, disease-free survival; LC, local control; DMFS, distant metastasis-free survival; ECOG, Eastern Cooperative Oncology Group; RT, radiotherapy; CCRT, concurrent chemoradiation.

them, chronic radiation fibrosis of grade 2 requiring treatment developed in two patients. Two other patients who had symptoms and diagnostic image of grade 2 chronic radiation pneumonitis consequently required admission care. Other than pneumonitis, grade 2 complications were as follows: 10 leucopenia, 2 thrombocytopenia, 2 anemia, and 11 esophagitis. Grade 3 leucopenia was seen in two patients, and one patient complained of grade 3 acute radiation esophagitis.

\section{Discussion and Conclusion}

Curative surgery still remains an indispensable treatment option for stages I to IIIA NSCLC. In completely resected stages II and IIIA NSCLC, PORT could be beneficial since the most common pattern of intrathoracic failure occurs along the surgical stump or in the regional lymph node [11]. Therefore, PORT is considered an adequate adjuvant therapy to improve LC, especially in N2 node positive patients and cases with residual disease. PORT Meta-analysis Trialists Group criticized the role of PORT in 1998, stating that PORT produced a 7\% detrimental effect in OS of all patients and no difference in OS in N2 node patients [12]. By contrast, several other studies have shown that PORT increased the locoregional control of N2 node positive patients $[4,6,8,13]$. Besides the 31 patients who had N2 nodes, our study also included $23 \mathrm{NO}, 13 \mathrm{N1}$, and $6 \mathrm{Nx}$ patients, but these patients required PORT due to involved or close resection margin and/or incomplete resection. In the Adjuvant Navelbine International Trialist Association (ANITA) trial, PORT improved survival in N1 node positive patients when given without chemotherapy. PORT improved survival of both observation and chemotherapy arm in N2 node positive patients [14]. The 5 -year OS was $46 \%$ in this study, comparable to results of other studies. The 2-year LC, 3-year DFS, and 3 -year DMFS rates were not inferior to those of previous studies. In this study, PORT was found to be a feasible and safe modality of adjuvant treatment in patients with multiple mediastinal nodes, close or involved margin, and/or residual disease.

Furthermore, although current evidence in the literature is limited this study tried to investigate whether CCRT had a further positive impact on the treatment outcome. The 5-year OS of CCRT arm was not inferior to RT arm despite more patients with higher stage were allocated in CCRT arm. This implies that CCRT could have contributed to better outcome in OS.

Adjuvant radiotherapy aids in sterilization of micrometastasis, which may lead to locoregional recurrence or distant metastasis. While studies investigating radiotherapy with a curative aim have reported that radiation dose escalation is associated with survival benefits, in the adjuvant setting, escalated radiation dose did not show improved LC or OS [15-17]. In contrast, radiation dose of more than 57.6 Gy showed improved DFS and DMFS in our study. CCRT may have caused such results because more patients with higher doses were in the CCRT arm. In order to determine the clear doserelationship of adjuvant radiotherapy, large scale randomized studies using current techniques, such as 3D-CRT, are needed. Adjuvant chemotherapy has a role in the prevention of 


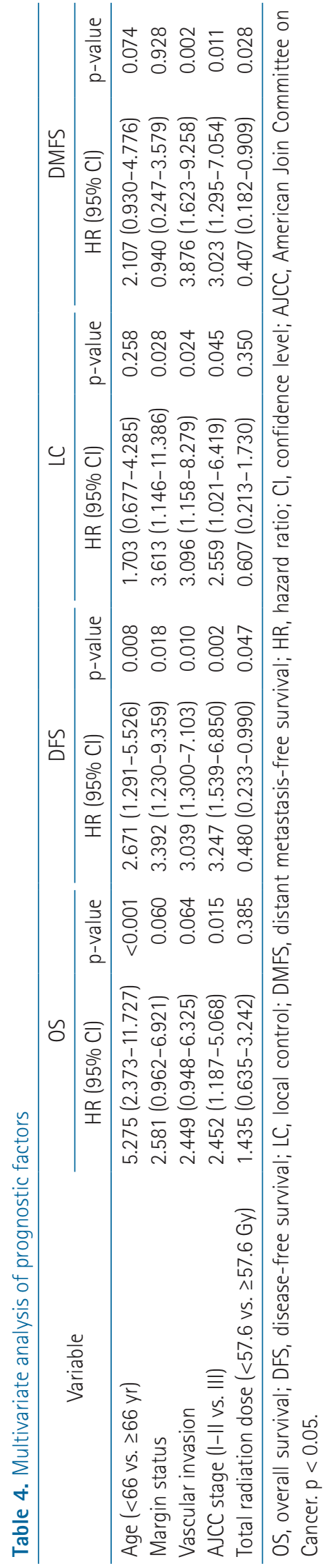

Table 5. Pattern of recurrence by the treatment arms

\begin{tabular}{lcccc}
\hline & $\begin{array}{c}\text { Total } \\
(\mathrm{n}=78)\end{array}$ & $\begin{array}{c}\text { RT } \\
(\mathrm{n}=48)\end{array}$ & $\begin{array}{c}\text { CCRT } \\
(\mathrm{n}=30)\end{array}$ & p-value \\
\hline Locoregional & $8(10.0)$ & $6(12.0)$ & $2(7.0)$ & - \\
Distant & $18(23.0)$ & $12(25.0)$ & $6(20.0)$ & - \\
$\begin{array}{l}\text { Locoregional \& } \\
\text { distant }\end{array}$ & $14(18.0)$ & $10(21.0)$ & $4(13.0)$ & - \\
Total & $40(51.0)$ & $28(58.0)$ & $12(40.0)$ & 0.433 \\
\hline
\end{tabular}

$\mathrm{RT}$, radiotherapy; CCRT, concurrent chemoradiation.

Table 6. Radiotherapy related toxicities by two treatment arms

\begin{tabular}{lccccc}
\hline & \multicolumn{2}{c}{ Grade 2} & & \multicolumn{2}{c}{ Grade 3 } \\
\cline { 2 - 3 } \cline { 5 - 6 } & RT & CCRT & & RT & CCRT \\
\hline Pneumonitis & & & & \\
$\quad$ Acute/Subacute & $2(2.6 \%)$ & $4(5.1 \%)$ & & - \\
$\quad$ Chronic & $1(1.3 \%)$ & $3(3.8 \%)$ & $1(1.3 \%)$ & $2(2.6 \%)$ \\
Others & & & & \\
$\quad$ Leucopenia & $3(3.8 \%)$ & $7(9.0 \%)$ & & $2(2.6 \%)$ \\
Thrombocytopenia & $1(1.3 \%)$ & $1(1.3 \%)$ & & - \\
Anemia & $1(1.3 \%)$ & $1(1.3 \%)$ & & - \\
$\quad$ Esophagitis & $5(6.4 \%)$ & $6(7.7 \%)$ & - & $1(1.3 \%)$ \\
\hline
\end{tabular}

Values are presented as number of patients (\%).

$\mathrm{RT}$, radiotherapy; CCRT, concurrent chemoradiation.

systemic failure caused by distant metastasis. In patients with completely resected NSCLC, adjuvant chemotherapy has been shown to improve survival of advanced stage (AJCC stage III) disease [18-20]. There was no statistical benefit in employing CCRT above RT. However, the survival rates of CCRT arm were all higher compared to those of RT alone arm in OS, DFS, LC, and DMFS. The propensity of improved results was more pronounced in DFS and LC as shown in Figs. 1 and 2, respectively. There are several reasons to explain the failure in extending the better survival of CCRT arm to statistical difference. Total number of patients as well as number of patients in each arm was too small to reflect the actual survival difference between the two arms. Another weakness of this study is the heterogeneity of chemotherapy regimen. More uniform regimen will eliminate the confounding effects caused by different drug potencies. There was no significantly more severe toxicity more observed in CCRT arm compared to RT alone arm. Further randomized trials need to be conducted to support the concept that CCRT should employ a current chemotherapy regimen in postoperative therapy.

Lung cancer patients are heavily affected by significant metabolic side effects. Hence, several patient-associated 
factors have been identified as clinically powerful prognostic factors. Stanley [21] stated that the 3 most important prognostic factors are initial performance status score, extent of disease, and weight loss in 6 months prior to diagnosis. In other studies, age, gender, and marital status have been found to be prognostic factors of survival [22]. In our study, patient age was the most important prognostic factor of OS, comparable to previous studies. Kim et al. [23] conducted retrospective analysis of PORT in stage III NSCLC. The 5-year DFS of patients with single station mediastinal lymph node metastasis was significantly better than that of multiple lymph node station (50\% vs. 8\%; $p=0.001$ ) [23]. Positive lymph nodes $>4$ was a significant prognostic factor of DFS in our study as well.

We acknowledge that this study has a number of limitations. Firstly, our study should be understood in the view of the inherent biases of a retrospective study design. The number of patients included in this study was relatively small from a single institution leading to low statistical power. There also may be a selection bias; patient and treatment related factors, such as age, performance status, pathologic stage, RT indication, and RT dose, were not evenly distributed in each treatment arm. Secondly, although indications of the treatment were apparent, variant stages of NSCLC from IA to IIIB were included in this study. The heterogeneous characteristics of the patients may have caused a confounding factor in statistical analysis.

Despite the limitations, this retrospective data demonstrated that CCRT after curative surgery is an effective treatment modality without causing severe toxicities. Although more patients with higher stage and more N2 disease were allocated, 3-year DFS and LC showed tendency of improvement in CCRT arm and 5-year OS of CCRT arm was not inferior to RT arm. So adequate scheduling of adjuvant therapy, including escalation of radiotherapy dose and concurrent administration of chemotherapy could be considered after surgery to improve survival among high-risk patients, such as multiple N2 disease following curative resection.

\section{Conflict of Interest}

No potential conflict of interest relevant to this article was reported.

\section{References}

1. Fry WA, Phillips JL, Menck HR. Ten-year survey of lung cancer treatment and survival in hospitals in the United States: a national cancer data base report. Cancer 1999;86:1867-76.

2. Trodella L, Granone $P$, Valente $S$, et al. Adjuvant radiotherapy in non-small cell lung cancer with pathological stage I: definitive results of a phase III randomized trial. Radiother Oncol 2002;62:11-9.

3. Harpole DH Jr, Herndon JE 2nd, Young WG Jr, Wolfe WG, Sabiston DC Jr. Stage I nonsmall cell lung cancer: a multivariate analysis of treatment methods and patterns of recurrence. Cancer 1995;76:787-96.

4. The Lung Cancer Study Group. Effects of postoperative mediastinal radiation on completely resected stage II and stage III epidermoid cancer of the lung. N Engl J Med 1986;315:1377-81.

5. Betticher DC, Hsu Schmitz SF, Totsch M, et al. Prognostic factors affecting long-term outcomes in patients with resected stage IIIA pN2 non-small-cell lung cancer: 5-year follow-up of a phase II study. Br J Cancer 2006;94:1099-106.

6. Stephens RJ, Girling DJ, Bleehen NM, Moghissi K, Yosef HM, Machin D. The role of post-operative radiotherapy in nonsmall-cell lung cancer: a multicenter randomised trial in patients with pathologically staged T1-2, N1-2, M0 disease. Medical Research Council Lung Cancer Working Party. Br J Cancer 1996;74:632-9.

7. Jung KW, Won YJ, Kong HJ, Oh CM, Seo HG, Lee JS. Cancer statistics in Korea: incidence, mortality, survival and prevalence in 2010. Cancer Res Treat 2013;45:1-14.

8. Lally BE, Zelterman D, Colasanto JM, Haffty BG, Detterbeck FC, Wilson LD. Postoperative radiotherapy for stage II or III nonsmall-cell lung cancer using the surveillance, epidemiology, and end results database. J Clin Oncol 2006;24:2998-3006.

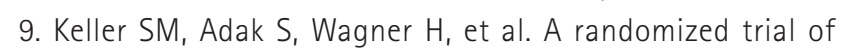
postoperative adjuvant therapy in patients with completely resected stage II or IIIA non-small-cell lung cancer. Eastern Cooperative Oncology Group. N Engl J Med 2000;343:121722.

10. Bradley JD, Paulus R, Graham MV, et al. Phase II trial of postoperative adjuvant paclitaxel/carboplatin and thoracic radiotherapy in resected stage II and IIIA non-small-cell lung cancer: promising long-term results of the Radiation Therapy Oncology Group (RTOG 9705). J Clin Oncol 2005;23:3480-7.

11. Logan DM, Lochrin CA, Darling G, Eady A, Newman TE, Evans WK. Adjuvant radiotherapy and chemotherapy for stage II or IIIA non-small-cell lung cancer after complete resection. Provincial Lung Cancer Disease Site Group. Cancer Prev Control 1997;1:366-78.

12. PORT Meta-analysis Trialists Group. Postoperative radiotherapy in non-small-cell lung cancer: systematic review and metaanalysis of individual patient data from nine randomised controlled trials. Lancet 1998;352:257-63. 
13. Sawyer TE, Bonner JA, Gould PM, et al. Effectiveness of postoperative irradiation in stage IIIA non-small cell lung cancer according to regression tree analyses of recurrence risks. Ann Thorac Surg 1997;64:1402-8.

14. Douillard JY, Rosell R, De Lena M, et al. Impact of postoperative radiation therapy on survival in patients with complete resection and stage I, II, or IIIA non-small-cell lung cancer treated with adjuvant chemotherapy: the adjuvant Navelbine International Trialist Association (ANITA) Randomized Trial. Int J Radiat Oncol Biol Phys 2008;72:695-701.

15. Socinski MA, Rosenman JG, Halle J, et al. Dose-escalating conformal thoracic radiation therapy with induction and concurrent carboplatin/paclitaxel in unresectable stage IIIA/ B nonsmall cell lung carcinoma: a modified phase I/II trial. Cancer 2001;92:1213-23.

16. Rosenman JG, Halle JS, Socinski MA, et al. High-dose conformal radiotherapy for treatment of stage IIIA/IIIB nonsmall-cell lung cancer: technical issues and results of a phase I/II trial. Int J Radiat Oncol Biol Phys 2002;54:348-56.

17. Kong FM, Ten Haken RK, Schipper MJ, et al. High-dose radiation improved local tumor control and overall survival in patients with inoperable/unresectable non-small-cell lung cancer: long-term results of a radiation dose escalation study. Int J Radiat Oncol Biol Phys 2005;63:324-33.

18. Arriagada R, Dunant $A$, Pignon JP, et al. Long-term results of the international adjuvant lung cancer trial evaluating adjuvant Cisplatin-based chemotherapy in resected lung cancer. J Clin Oncol 2010;28:35-42.

19. Winton $T$, Livingston $R$, Johnson $D$, et al. Vinorelbine plus cisplatin vs. observation in resected non-small-cell lung cancer. N Engl J Med 2005;352:2589-97.

20. Butts CA, Ding $K$, Seymour $L$, et al. Randomized phase III trial of vinorelbine plus cisplatin compared with observation in completely resected stage IB and II non-small-cell lung cancer: updated survival analysis of JBR-10. J Clin Oncol 2010;28:29-34.

21. Stanley KE. Prognostic factors for survival in patients with inoperable lung cancer. J Natl Cancer Inst 1980;65:25-32.

22. Buccheri G, Ferrigno D. Prognostic factors in lung cancer: tables and comments. Eur Respir J 1994;7:1350-64.

23. Kim MY, Wu HG, Kim HJ, et al. Role of postoperative radiotherapy for patients with pathological stage III non-small-cell lung cancer after curative resection. J Korean Soc Ther Radiol Oncol 2011;29:44-52. 